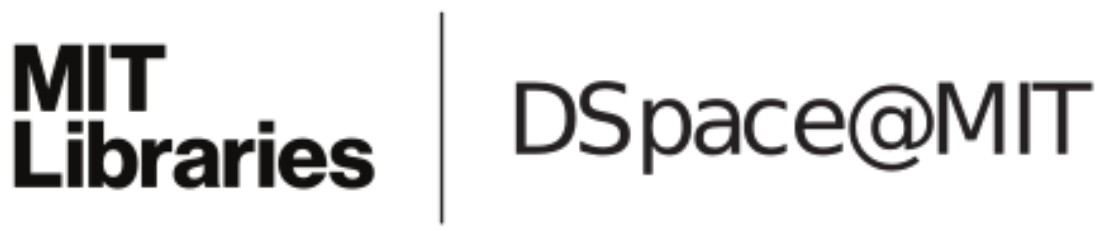

\author{
MIT Open Access Articles
}

\begin{abstract}
Preparation of reduced-quantumuncertainty input states for an atomic clock
\end{abstract}

The MIT Faculty has made this article openly available. Please share how this access benefits you. Your story matters.

Citation: Schleier-Smith, M. H., I. D. Leroux, and V. Vuletic. "Preparation of reduced-quantumuncertainty input states for an atomic clock." Time and Frequency Metrology II. Ed. Tetsuya Ido \& Derryck T. Reid. San Diego, CA, USA: SPIE, 2009. 743107-10. @ 2009 SPIE

As Published: http://dx.doi.org/10.1117/12.828171

Publisher: Society of Photo-optical Instrumentation Engineers

Persistent URL: http://hdl.handle.net/1721.1/52734

Version: Final published version: final published article, as it appeared in a journal, conference proceedings, or other formally published context

Terms of Use: Article is made available in accordance with the publisher's policy and may be subject to US copyright law. Please refer to the publisher's site for terms of use. 


\title{
Preparation of Reduced-Quantum-Uncertainty Input States for an Atomic Clock
}

\author{
M. H. Schleier-Smith, I. D. Leroux, and V. Vuletić \\ Department of Physics, MIT-Harvard Center for Ultracold Atoms, \\ and Research Laboratory of Electronics, \\ Massachusetts Institute of Technology, Cambridge, Massachusetts 02139, USA
}

\begin{abstract}
Atomic clocks have reached the Standard Quantum Limit (SQL) of precision, ${ }^{1}$ set by the projection noise inherent in measurements on uncorrelated atoms. It is possible to overcome this limit by entangling the atoms to generate a "squeezed state" of the atomic ensemble. We use the collective interaction of an atomic ensemble with a far-detuned light field in an optical resonator to prepare squeezed states by two different methods: quantum non-demolition (QND) measurement and Hamiltonian evolution. We apply both methods to an ensemble of $5 \times 10^{4}{ }^{87} \mathrm{Rb}$ atoms in a superposition of hyperfine clock states. We measure the suppression of projection noise and compare it to the accompanying reduction in signal, thereby quantifying the net gain in spectroscopic sensitivity.

By QND measurement, with resolution up to $9 \mathrm{~dB}$ below the projection noise level, we achieve 3.0(8) $\mathrm{dB}$ of metrologically relevant squeezing. Whereas the measurement-based approach relies on knowledge of the (randomly distributed) measurement outcome to produce a conditionally squeezed state, the method of Hamiltonian evolution produces a known squeezed state independent of detector performance. We mimic the dynamics of the one-axis twisting Hamiltonian, proposed as a generator of squeezed states by Kitagawa and Ueda, ${ }^{2}$ by using the atom-induced frequency shift of the resonator mode and the corresponding resonator-field-induced shift of the atomic transition frequency to introduce an effective interaction among the atoms. The resulting deterministic squeezing is sufficient to allow a 6.0(4) dB improvement in spectroscopic sensitivity over the SQL.
\end{abstract}

Keywords: metrology, atomic clock, spin squeezing, entanglement, Standard Quantum Limit

\section{INTRODUCTION}

In the past decade, the precision of measurement devices ranging from microwave ${ }^{1}$ and optical ${ }^{3-5}$ atomic clocks to atom interferometers ${ }^{6}$ has approached the Standard Quantum Limit (SQL). ${ }^{7,8}$ All of these devices employ a Ramsey sequence, in which a $\pi / 2$ pulse places an initially optically pumped ensemble of $N_{0}$ two-level atomsabstractly, spin-1/2 particles - into a symmetric superposition state with phase uncertainty $\Delta \phi_{\text {in }}=1 / \sqrt{N_{0}}$. After allowing this coherent spin state (CSS) to precess for some time, the final phase is read out by converting it via a second $\pi / 2$ pulse into a population difference between the two clock levels. Even if this population difference is read out perfectly, the phase uncertainty $\Delta \phi_{\text {in }}$ of the initial coherent spin state (CSS) results in the quantum-limited uncertainty $1 / \sqrt{N_{0}}$ in the phase accumulated during the precession.

A general state of the ensemble of $N_{0}$ atoms with levels $|\uparrow\rangle=\left|s_{z}=+1 / 2\right\rangle$ and $|\downarrow\rangle=\left|s_{z}=-1 / 2\right\rangle$ is characterized by a total ensemble spin $\mathbf{S}$ with $S \leq N_{0} / 2$, where the equality holds for symmetric states of the ensemble. For a given $\langle\mathbf{S}\rangle=S \hat{x}$-where we have assumed an orientation along the $\hat{x}$-axis - the minimum-uncertainty uncorrelated state is the CSS, with phase uncertainty $1 / \sqrt{2 S}$ corresponding to a spin variance $\left(\Delta S_{y}\right)^{2}=S / 2$. Achieving precision beyond the SQL requires that, at the beginning of the Ramsey precession, the ensemble be in an entangled state such that $\left(\Delta S_{y}\right)^{2}<S / 2$ : a spin-squeezed ${ }^{2,9-21}$ (specifically, phase-squeezed) state. Figure 1 illustrates the preparation of a generic spin-squeezed state $(\mathbf{C})$, characterized by an uncertainty less than the CSS uncertainty in some direction transverse to $\hat{x}$; and the use of this state in a modified Ramsey

Send correspondence to M.H.S.-S.: E-mail: schleier@mit.edu, Telephone: 16174523578

Time and Frequency Metrology II, edited by Tetsuya Ido, Derryck T. Reid, Proc. of SPIE Vol. 7431, $743107 \cdot$ @ 2009 SPIE · CCC code: 0277-786X/09/\$18 · doi: 10.1117/12.828171 


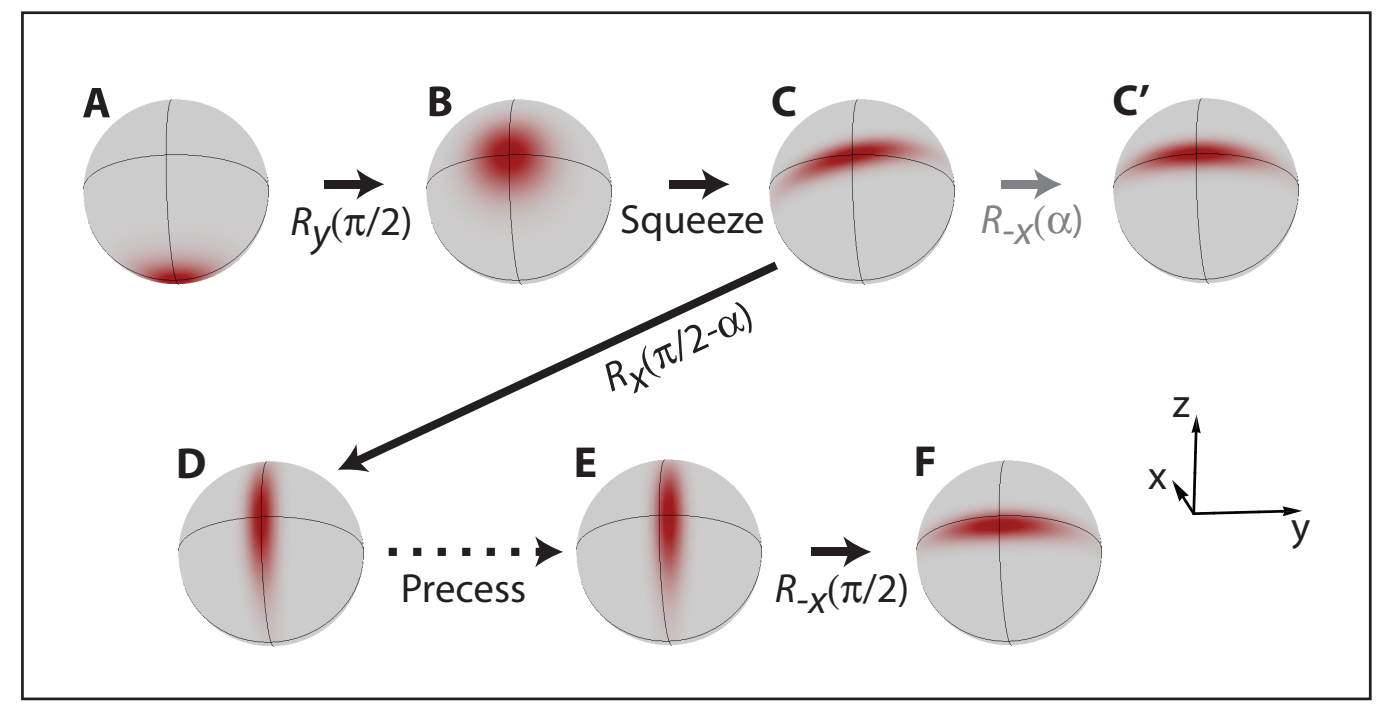

Figure 1. Modified Ramsey sequence incorporating spin squeezing. Initially, the ensemble is optically pumped into one clock state $|\downarrow\rangle$ (A). A $\pi / 2$ pulse places the ensemble in a CSS superposition of states $|\downarrow\rangle$ and $|\uparrow\rangle$ B. The CSS is converted into a state which is squeezed along some direction, at an angle $\alpha$ from the z-axis $(\mathbf{C})$. The rotation of the squeezed state into a phase-squeezed state $(\mathbf{D})$ marks the beginning of the free precession of the clock. A final $\pi / 2$ pulse converts the accumulated phase $(\mathbf{E})$ into a population difference $(\mathbf{F})$, the variance of which is reduced relative to the CSS variance. In the present work, we verify spin squeezing by directly rotating the state $\mathbf{C}$ into the number-squeezed state $\mathbf{C}$, so as not to introduce extra noise via the Ramsey sequence. Note that in the case of conditional squeezing by measurement of $S_{z}$, $\alpha=0$.

sequence, initiated by a rotation that places the squeezing in the phase quadrature (D). The preparation of such spin-squeezed input states $\mathbf{C}$ is the focus of this work.

We characterize the spin noise of each prepared state by measuring the variance of the population difference $N_{\uparrow}-N_{\downarrow}=2 S_{z}$ between clock states after a suitable rotation about $\hat{x}$ (Fig. $1 \mathbf{C}^{\prime}$ ). Since any squeezing process not only reduces $\left(\Delta S_{z}\right)^{2}$ but also shortens the mean ensemble spin vector $\langle\mathbf{S}\rangle$ relative to its length $S_{\text {in }}$ in the CSS, ${ }^{9}$ the squared signal-to-noise ratio $|\langle\mathbf{S}\rangle|^{2} /\left(\Delta S_{z}\right)^{2}$ must be compared to its value $2 S_{\text {in }}$ in the CSS in order to quantify metrological gain. A metrology factor $\zeta_{\mathrm{m}} \equiv 2 S_{\mathrm{in}}\left(\Delta S_{z}\right)^{2} /|\langle\mathbf{S}\rangle|^{2}<1$ corresponds to metrologically relevant spin squeezing.

Experimental realizations of spin squeezing to date are few but nevertheless encompass a wide spectrum of atomic systems. Up to three ions have been entangled to achieve spectroscopy beyond the SQL. ${ }^{10,22} \mathrm{~A}$ Bose Einstein condensate in a multiple-well potential can evolve from a CSS into a spin-squeezed state under the influence of collisional interactions. ${ }^{20,21}$ To induce spin squeezing in a dilute atomic ensemble, atom-light interaction can substitute for direct interatomic interaction. ${ }^{9,12-14,16,18,19,23,24}$ To date, the most effective approach to such light-induced squeezing has been to perform, via the light field, a quantum non-demolition (QND) measurement that projects the atomic ensemble into an entangled state. ${ }^{18,19}$ This state is conditionally squeezed, in the sense that the particular state prepared depends upon the measurement outcome.

In principle, atom-light interactions can also produce deterministic spin squeezing if the light passes through the atomic sample multiple times so as to apply feedback. ${ }^{15,25,26}$ Here, we introduce a simple approach to such deterministic spin squeezing which exploits the Hamiltonian dynamics of an atomic ensemble interacting with a light field in an optical resonator. We demonstrate this dynamic squeezing on the $\left|F=1, m_{F}=0\right\rangle \rightarrow$ $\left|F=2, m_{f}=0\right\rangle$ clock transition of ${ }^{87} \mathrm{Rb}$ and compare it with the conditional squeezing induced by resonatorenhanced QND measurement in the same system. 


\section{THEORETICAL OVERVIEW}

Figure 2(a) illustrates the level scheme for our light-induced squeezing. A resonator mode is placed (in an idealized 3-level system) at equal and opposite detunings $\pm \delta$ from transitions $|\uparrow\rangle \rightarrow|e\rangle$ and $|\downarrow\rangle \rightarrow|e\rangle$ associated with the two clock states. Thus, the dipole interaction of the atom with the intracavity light has equal magnitude - but opposite sign - for atoms in the two clock states. The detuning is much larger than the atomic excited-state linewidth, $\delta \gg \Gamma$, so that the interaction Hamiltonian is given by

$$
H_{I} / \hbar=\hat{a}^{\dagger} \hat{a}\left(\frac{g^{2}}{\delta} N_{\uparrow}-\frac{g^{2}}{\delta} N_{\downarrow}\right)=2 \frac{g^{2}}{\delta} \hat{a}^{\dagger} \hat{a} \hat{S}_{z},
$$

where $2 g$ is the vacuum Rabi frequency and $\hat{a}^{\dagger} \hat{a}$ represents the intracavity photon number. The atom-light interaction has two complementary manifestations: a frequency shift of the resonator mode by $\left(2 g^{2} / \delta\right) S_{z}$; and, via the AC Stark shift on the atomic levels, a phase shift between the clock states averaging $4 g^{2} /(\delta \kappa)$ per photon transmitted through the resonator. The same dimensionless parameter $\phi_{1} \equiv 4 g^{2} /(\delta \kappa)$ characterizes both the atomic phase shift per photon and the resonator mode shift $\left(\phi_{1} / 2\right) \kappa S_{z}$.

A probe laser is placed at a detuning $\kappa / 2$ from cavity resonance so that, for $S_{z} \phi_{1} \ll 1$, the intracavity and transmitted power depend linearly on $S_{z}$. A measurement of the transmitted probe power is thus a QND measurement of $S_{z}$; conditioned upon the outcome of this measurement, the variance $\left(\Delta S_{z}\right)^{2}$ is reduced.

This same configuration also allows a second route to squeezing. The dependence of intracavity probe photon number $\hat{a}^{\dagger} \hat{a}$ on $S_{z}$ yields an atomic phase shift that is linear in $S_{z}$, which induces a twisting of the Bloch sphere similar to the dynamics of the "one-axis twisting" Hamiltonian" ${ }^{26} H \propto S_{z}^{2}$. The twisting has no effect on the variance of $S_{z}$ but squeezes the state along an oblique axis, as illustrated in Fig. 2(b).

The evolution of the squeezed state from an initial CSS can be understood, in a simple semiclassical model, by evaluating the phase shift induced on each atom by the intracavity light for a given net ensemble spin $S_{z}$ and thereby obtaining the mean and variance of $S_{y}$ given $S_{z} \cdot{ }^{27}$ Key results from this model are as follows:

- Neglecting technical noise and decoherence, the elliptical geometry of the squeezed state is described by a single "shearing parameter" $Q=S p \phi_{1}^{2}$, where $p$ is the number of photons transmitted through the resonator

- The variance along the minor axis decreases as $\left(\Delta S_{z}\right)_{\min }^{2} \approx 1 /(1+Q)$ (until reaching a limit due to either the curvature of the Bloch sphere or photon scattering)

- The orientation of the minor axis asymptotically approaches the $\hat{z}$-direction with increasing shearing

- The uncertainty product $\Delta S_{z \min } \Delta S_{z \max }$ grows in proportion to $\sqrt{Q}$ due to phase broadening induced by photon shot noise in the intracavity power

\section{EXPERIMENTAL SETUP}

We describe here the experimental setup used for dynamic squeezing; the conditional squeezing data were taken earlier in the same setup, ${ }^{19}$ except for minor subsequent technical improvements. The atoms are trapped in the mode of a near-confocal optical resonator using a standing-wave optical dipole trap of $851 \mathrm{~nm}$ wavelength. To minimize inhomogeneous broadening of the clock transition by the optical trap, we use a combination of elliptical polarization and magnetic bias field which yields a first-order cancellation of the scalar and vector AC Stark shifts (see Appendix A).

We probe (and squeeze) via the optical resonator on the $D_{2}$ line, using linearly polarized $780 \mathrm{~nm}$ light. At this wavelength, the resonator has a finesse of $5.6(2) \times 10^{3}$, a linewidth $\kappa=2 \pi \times 1.01(3) \mathrm{MHz}$, and a mode waist size of 56.9(4) $\mu \mathrm{m}$ at the position of the atoms. The coupling of atoms to the probe light is maximally inhomogeneous because the standing wave formed by the probe light is incommensurate with that of the trap (Fig. 2(c)). The inhomogeneously coupled ensemble of $N_{\mathrm{a}} \leq 5 \times 10^{4}$ atoms is equivalent to an ensemble of $N_{0}=\left(\langle\eta\rangle_{e}^{2} /\left\langle\eta^{2}\right\rangle_{e}\right) N_{\mathrm{a}} \approx 0.66 N_{\mathrm{a}}$ atoms uniformly coupled to the resonator with effective single-atom cooperativity $\eta_{\mathrm{eff}}=\left\langle\eta^{2}\right\rangle_{e} /\langle\eta\rangle_{e}=0.145(5),{ }^{19}$ where \langle\rangle$_{e}$ denotes an average over the atomic ensemble and $\eta(\mathbf{r})=4 g_{0}^{2} /(\kappa \Gamma)$ in 


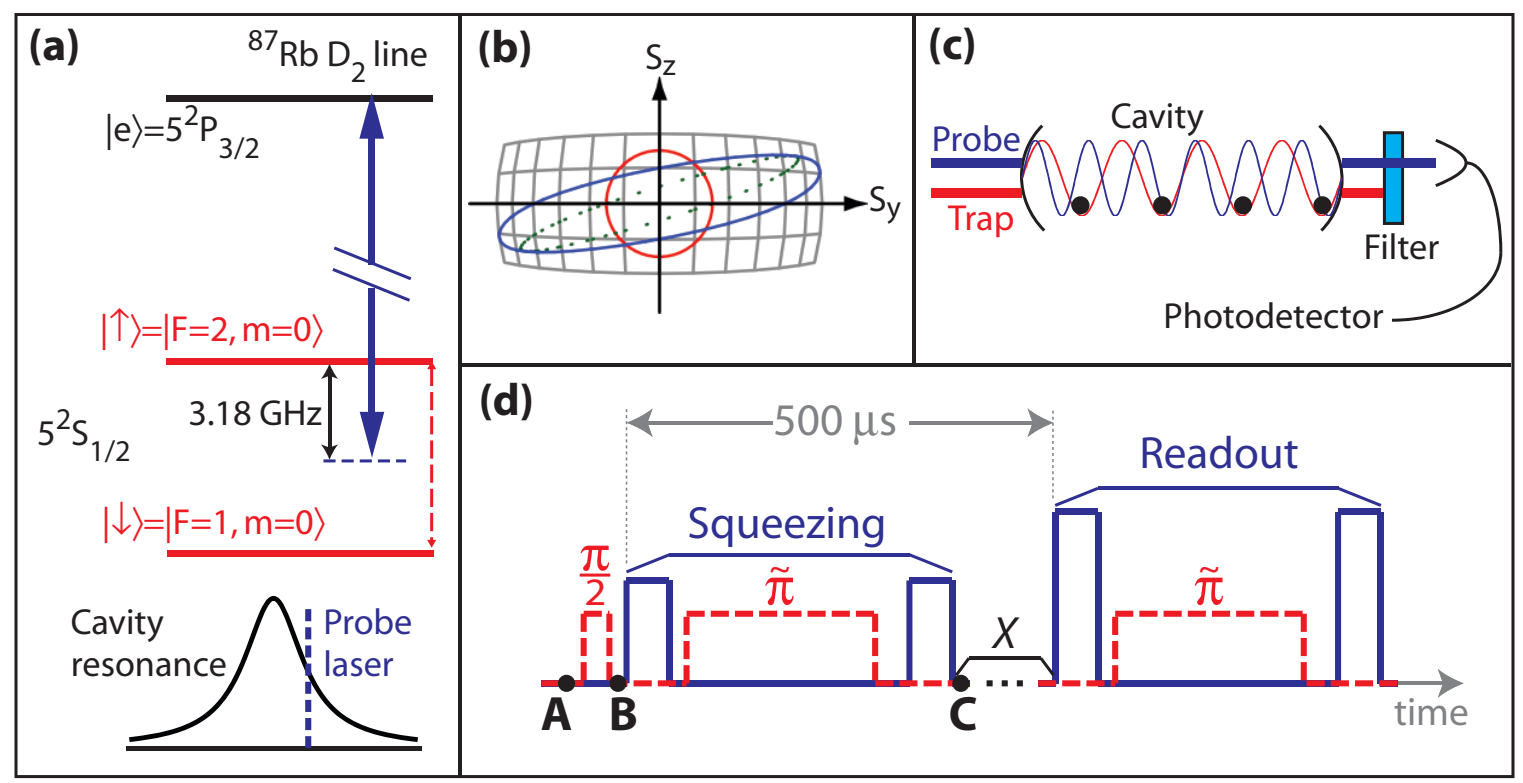

Figure 2. Experimental setup and sequence. (a) Atomic level structure and tuning of probe laser relative to atomic transitions and cavity resonance. (b) Shearing of a coherent state (red circle) into a squeezed state (blue ellipse) under the scheme depicted in (a). The evolution is similar to the "one-axis twisting" dynamics of an interaction Hamiltonian $H_{I} \propto S_{z}^{2}$ (dotted green ellipse), but in our system the state is broadened by photon shot noise. (c) Schematic of probe and trap light in optical resonator. (d) Timing of probe pulses (solid blue line) and microwave pulses (dashed red line) in preparation and readout of a squeezed state. $\tilde{\pi}$ designates a $\operatorname{SCROFULOUS}^{28} \pi$ pulse. Points A-C indicate the state of the ensemble as depicted in Fig. 1. Various procedures are inserted at $X$ to measure the noise of a spin component other than $S_{z}$, evaluate the interference contrast, or operate a clock, as described in the text.

terms of the coupling $g_{0}$ of a two-level atom to the resonator. In our system, $g^{2}$ is reduced by a factor of $2 / 3$, the oscillator strength of the $D_{2}$ line. Thus, the (appropriately averaged) atomic phase shift per photon transmitted through the resonator is $\phi_{1}=(2 / 3) \eta_{\mathrm{eff}} \Gamma / \delta=1.78(6) \times 10^{-4} \mathrm{rad}$; and the resonator mode shift per effective atom of population difference $N_{\uparrow}-N_{\downarrow}=2 S_{z}$ is $\kappa \phi_{1} / 4=4.5(2) \times 10^{-5} \kappa$.

The TEM00 resonator mode used for probing is tuned 3.18(1) $\mathrm{GHz}$ to the blue of the $\left|5^{2} S_{1 / 2}, F=2\right\rangle \rightarrow$ $\left|5^{2} P_{3 / 2}, F^{\prime}=3\right\rangle$ transition. The probe light placed at detuning $\kappa / 2$ from this resonance is a sideband modulated at $36 \mathrm{GHz}$ onto a laser that is locked via the Pound-Drever-Hall technique ${ }^{29}$ to a TEM01 mode of the resonator. We always apply probe light in two $50 \mu$ s pulses separated by $330 \mu$ d during which we apply a composite (SCROFULOUS $\left.{ }^{28}\right) \pi$ pulse to allow a spin-echo cancellation of inhomogeneous broadening.

Figure 2(d) shows the squeezed-state preparation and readout sequence. To measure the mean and variance of an ensemble spin vector, we repeat this sequence 100 times in 10 sets of 10 repetitions with the same loaded atoms.

\section{DATA}

\subsection{Calibration of Projection Noise}

In order to quantify spin squeezing, we require an accurate calibration of the quantum projection noise $\left(\Delta S_{z}\right)_{\text {CSS }}^{2}$ of the CSS. Figure 3 shows two measurements of the CSS variance as a function of effective atom number $N_{0}$ which are essentially equivalent but sensitive to different technical noise sources. The open red triangles give the variance $(\delta N)^{2}=4\left(\delta S_{z}\right)^{2}$ of a measurement of $S_{z}$ following the preparation of a CSS along $\hat{x}$ (the squeezing measurement in Fig. 2(d)) in effective atom units. The open red circles are obtained by preparing a CSS twice from the same loaded atoms, measuring $S_{z}$ in each case, and evaluating half the variance of the difference between the two measurements. The dashed red curve indicates the projection noise level as determined from the atom 


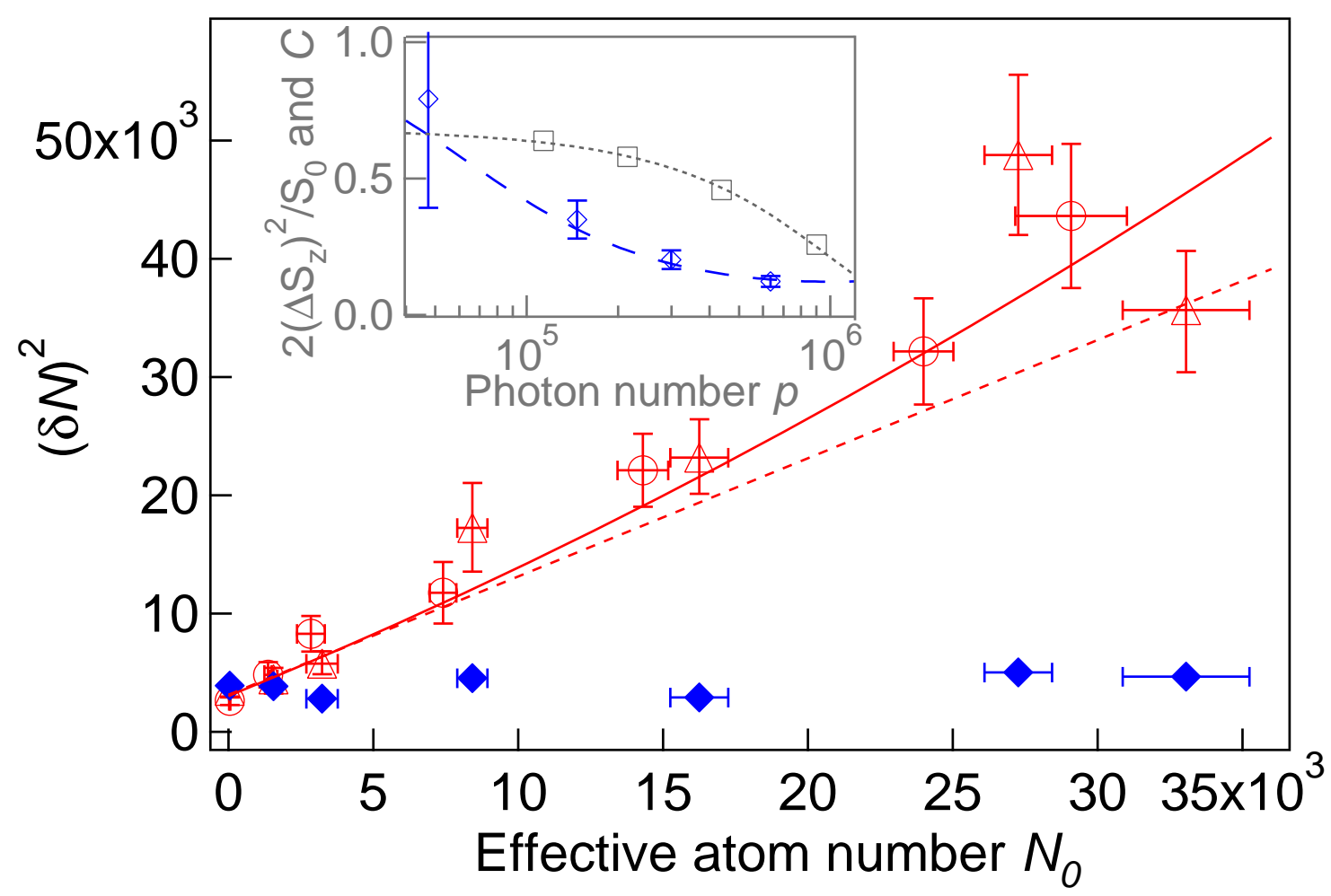

Figure 3. Spin measurements performed using $p=5 \times 10^{5}$ probe photons to characterize CSS projection noise and measurement resolution. Open red triangles: measured variance of $N=2 S_{z}$ after preparation of a CSS in the $x y$ plane. Open red circles: half the variance of the difference between measurements of $S_{z}$ following two independent CSS preparations. The red data are consistent with the variance expected from the measured atom number (dashed red line) plus smaller contributions from technical noise of the CSS preparation, including drifts in microwave power (solid red curve). Solid blue diamonds: measurement resolution $(\delta N)^{2}=4\left(\delta S_{z}\right)^{2}$, given by half the variance of the difference between two successive measurements of $S_{z}$ following a single CSS preparation. Inset: Normalized spin noise $\left(\Delta S_{z}\right)^{2} /\left(\Delta S_{z}\right)_{\text {CSS }}^{2}$ (solid blue diamonds) vs photon number $p$ at fixed atom number $N_{0}=3.3(2) \times 10^{4}$. The reduction of normalized spin noise with increasing photon number occurs at the cost of decreasing contrast $\mathcal{C}=|\langle\mathbf{S}\rangle| / S_{0}($ open gray squares).

number, measured from the resonator shifts induced by optically pumping the ensemble into each of the two clock states in turn. The data are consistent with the projection noise expected for the measured atom number augmented by smaller technical noise contributions (solid red curve), which we attribute to drifts in microwave power and to an effect of leakage light.

In quantifying spin squeezing, we always normalize to the true CSS variance $\left(\Delta S_{z}\right)_{\text {CSS }}^{2}=N_{0} / 4$ determined from the measured atom number, not to the larger technical noise. We have an accurate calibration of the resonator mode shift per atom $\kappa \phi_{1} / 4$ from measured resonator parameters, and we have independently confirmed it by directly measuring the atomic phase shift $\phi_{1} \cdot{ }^{19}$

\subsection{Normalized Spin Noise}

\subsubsection{Conditional Noise Reduction via QND Measurement}

To quantify the spin noise conditioned on the squeezing measurement, we perform an identical readout measurement following the squeezing measurement. Although the unconditional variance $\left(\delta S_{z}\right)_{U}^{2}$ of each measurement is at least as great as the CSS variance, the information from the squeezing measurement helps to predict the 


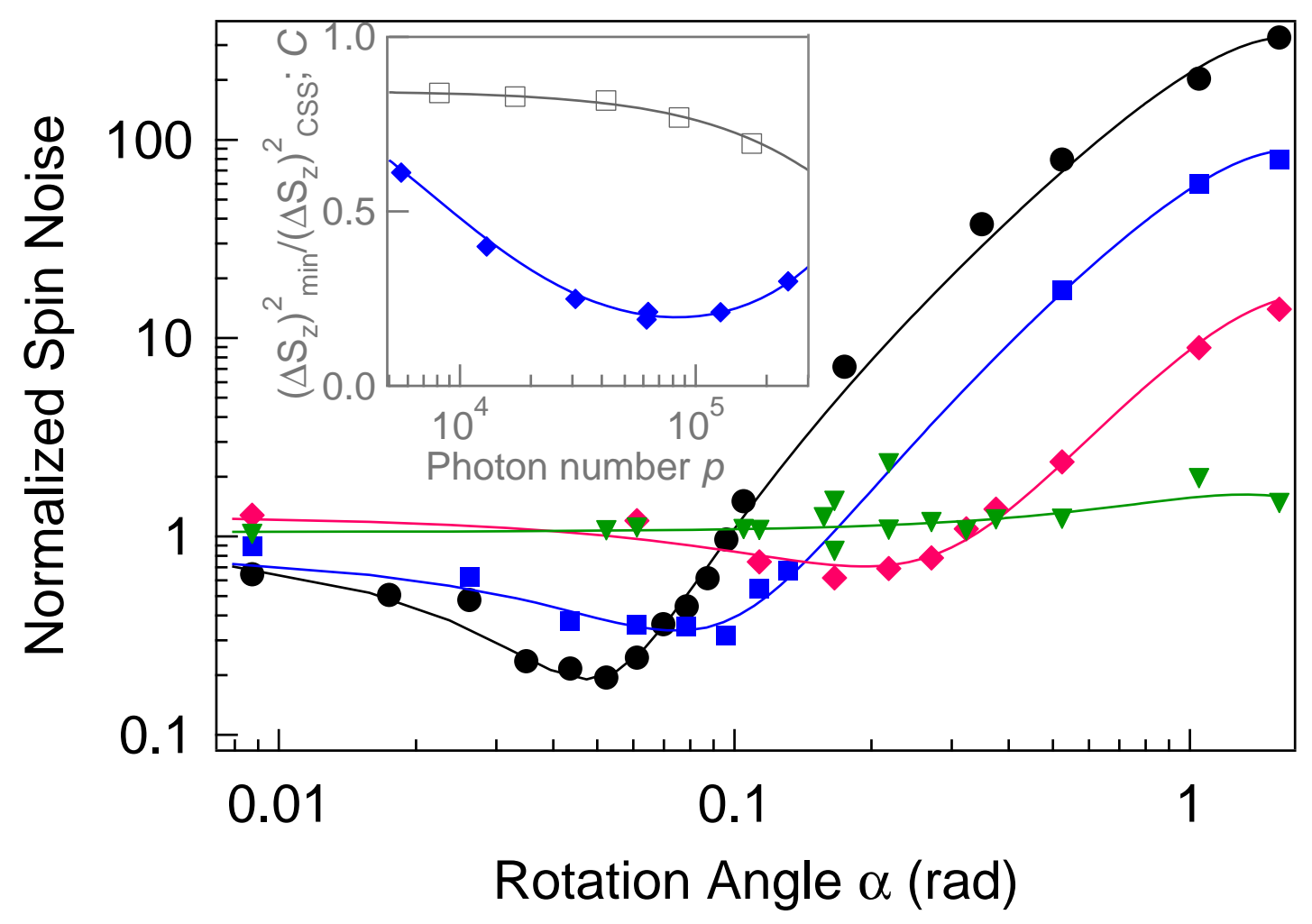

Figure 4. Normalized spin noise $\left(\Delta S_{z}\right)^{2} /\left(\Delta S_{z}\right)_{\text {CSS }}^{2}$ measured after rotating by a variable angle about $\langle\mathbf{S}\rangle$ four different states corresponding to shearing parameters $Q=0$ (green triangles), $Q=4.5$ (magenta diamonds), $Q=15$ (blue squares) and $Q=28$ (black circles). With increasing $Q$, the angle of minimum spin noise decreases, while the uncertainty product $\Delta S_{z \min } \Delta S_{z \max }$ increases. Inset: dependence on photon number $p$ of minimum normalized spin noise $\left(\Delta S_{z}\right)_{\min }^{2} /\left(\Delta S_{z}\right)_{\mathrm{CSS}}^{2}$ (solid blue diamonds) at fixed atom number $N_{0}=28000(1000)$; and of contrast $\mathcal{C}$ (open gray squares).

readout with reduced uncertainty. Since the squeezing and readout measurements are identical and uncorrelated, the resolution $\left(\delta S_{z}\right)_{M}^{2}$ of each such measurement is given by half the variance of the difference between the measurements. The squeezing measurement reduces the uncertainty of an ideal, noiseless readout of $S_{z}$ from $\left(\delta S_{z}\right)_{U}^{2}-\left(\delta S_{z}\right)_{M}^{2}$ to $\left(\Delta S_{z}\right)^{2}=\left(\delta S_{z}\right)_{M}^{2}\left(\left(\delta S_{z}\right)_{U}^{2}-\left(\delta S_{z}\right)_{M}^{2}\right) /\left(\delta S_{z}\right)_{U}^{2}{ }^{19}$ We plot the normalized spin noise $\left(\Delta S_{z}\right)^{2} /\left(\Delta S_{z}\right)_{\text {CSS }}^{2}$ in the inset to Fig. 3 as a function of the number $p$ of probe photons used in each measurement and find that it reaches $9.2(8) \mathrm{dB}$ at $p=1.2 \times 10^{6}$.

\subsubsection{Deterministic Noise Reduction via Hamiltonian Dynamics}

To observe the dynamic squeezing, we insert between squeezing and readout a rotation about the $x$-axis by a variable angle and evaluate the dependence of variance $\left(\delta S_{z}\right)^{2}$ of the readout measurement on rotation angle. We always use $1.1(2) \times 10^{6}$ photons for the readout measurement so that the variance contributed by the technical noise of the readout (equivalent to the projection noise of 900 atoms) is less than the spin noise even after appreciable squeezing. We conservatively estimate the true spin noise as $\left(\Delta S_{z}\right)^{2} \approx\left(\delta S_{z}\right)^{2}$. Figure 4 shows four representative curves of normalized spin noise $\left(\Delta S_{z}\right)^{2} /\left(\Delta S_{z}\right)_{\text {CSS }}^{2}$ as a function of rotation angle for different values of the shearing parameter $Q \equiv S p \phi_{1}^{2}$. From the sinusoidal fits to these data, we extract the minimum spin noise $\left(\Delta S_{z}\right)_{\min }^{2}$, the uncertainty product $\Delta S_{z_{\min }} \Delta S_{z \max }$, and the angle $\alpha$ of the minor axis of the squeezed state relative to the $z$-axis, finding these quantitites in good agreement with a model including known technical effects. $^{27}$ We plot the normalized spin noise $\left(\Delta S_{z}\right)_{\min }^{2} /\left(\Delta S_{z}\right)_{\mathrm{CSS}}^{2}$ in the inset to Fig. 4. 


\subsection{Interference Contrast}

In order to quantify spin squeezing, we must not only measure spin noise but also account for the reduction in interference contrast $\mathcal{C}=S / S_{0}$ due to the squeezing light. We measure the contrast by either driving Rabi oscillations or inserting a Ramsey sequence between the squeezing and readout measurements (at $X$ in Fig. $2(\mathrm{~d}))$. The inset to Fig. 3 shows the contrast of Rabi oscillations measured at $N_{0}=4.0(1) \times 10^{3}$ as a function of squeezing photon number under the experimental conditions of the conditional squeezing. Note that at larger atom number, the "one-axis twisting" dynamics in our system lead to an appreciable $S_{z}$-dependent phase. This phase fluctuates from shot to shot but can be predicted based on the outcome of the squeezing measurement. Thus, the result of the squeezing measurement must be used not only to benefit from the conditionally reduced noise in $S_{z}$ but also to achieve the full contrast measured at smaller atom number.

The inset to Fig. 4 shows the contrast of Ramsey fringes measured under the experimental conditions of the dynamic squeezing. The initial contrast $\mathcal{C}_{\text {in }}$ is higher than that measured under the conditional squeezing conditions because the resonator locking light is further detuned.

\section{RESULTS}

In Fig. 5, we compare the metrology factors $\zeta_{\mathrm{m}}=2\left(\Delta S_{z}\right)^{2} S_{\text {in }} /|\langle\mathbf{S}\rangle|^{2}$ obtained by the measurement-induced and dynamic spin squeezing as a function of photon number $p$ at similar atom numbers, with $S_{\text {in }}=12000(1000)$ for both datasets. The dynamic squeezing commences at lower photon number because its performance is more nearly photon shot noise limited. In particular, the dynamic squeezing performs ideally when the fluctuations in intracavity power are dominated by photon shot noise. This condition holds in our system for $p<5 \times 10^{4}$; at larger photon number, the dominant noise is contributed by laser frequency jitter of $1.6(4) \times 10^{-3} \kappa$ between the two 50us probe pulses which induce the squeezing. (Noise common to the two pulses is removed by the spin echo.) The conditional squeezing, however, always suffers from the $43(4) \%$ quantum efficiency of our detection and a factor of 2 in excess noise of the avalanche photodetector.*

Both methods of squeezing improve with increasing photon number until encountering technical limitations. The conditional squeezing is limited by the reduction in contrast associated with the inhomogeneous coupling of atoms to probe light; the rephasing by spin echo is imperfect because of atomic motion. The dynamic squeezing is limited not by contrast loss but by an increase in spin noise $\left(\Delta S_{z}\right)_{\min }^{2}$ at large photon number (and more generally, at large $Q$ ). Such an effect is expected for shearing parameters greater than $Q_{c} \sim \sqrt{N_{0}}$ due to the curvature of the Bloch sphere, but its onset in our system occurs at somewhat smaller $Q$. This may be due to the inhomogeneous atomic phase induced by the resonator locking light - evidenced by the finite initial contrast $\mathcal{C}_{\text {in. }}$. Such a phase inhomogeneity can make some atoms sample the curvature of the Bloch sphere at reduced $Q_{c}$.

Because of the adverse effects arising with increasing photon number, the dynamic squeezing - which allows the greater noise reduction at low photon number - is more effective. Both conditional and dynamic squeezing can be improved by cooling the atoms further and by modifying the laser system to lock to the resonator with further-detuned light (to minimize contrast loss) at higher power (to improve the frequency stabilization). A rough estimate for the fundamental limit posed by photon scattering ${ }^{14,15,24}$ is, for the dynamic squeezing, $\zeta_{\mathrm{m}}^{-1}=\sqrt{N_{0} \eta_{\text {eff }} / 6}=14 \mathrm{~dB}$ in our system with collective cooperativity $N_{0} \eta=4000$. This estimate is probably a conservative one, as it does not take into account the continual squeezing of the noise added by photon scattering.

\section{CONCLUSION}

We have applied two different approaches to spin squeezing on the $\left|F=1, m_{F}=0\right\rangle \rightarrow\left|F=2, m_{F}=0\right\rangle$ clock transition of ${ }^{87} \mathrm{Rb}$, both harnessing the collective interaction of an atomic ensemble with light in an optical resonator. By a QND measurement on the light, we achieve 3.0(8) dB of metrologically relevant conditional spin squeezing. The more powerful approach exploits the "one-axis twisting" dynamics of the atom-light interaction to

*We reduce the sensitivity of the conditional squeezing to laser frequency jitter by placing a "compensation" sideband of the probe laser at detuning $-\kappa / 2$ relative to a cavity mode far detuned from the atomic transitions. ${ }^{19}$ However, this sideband also doubles the photon shot noise on the detector. At the photon number where we find the minimum metrology factor, this compensation sideband may even add slightly more noise than it removes. 


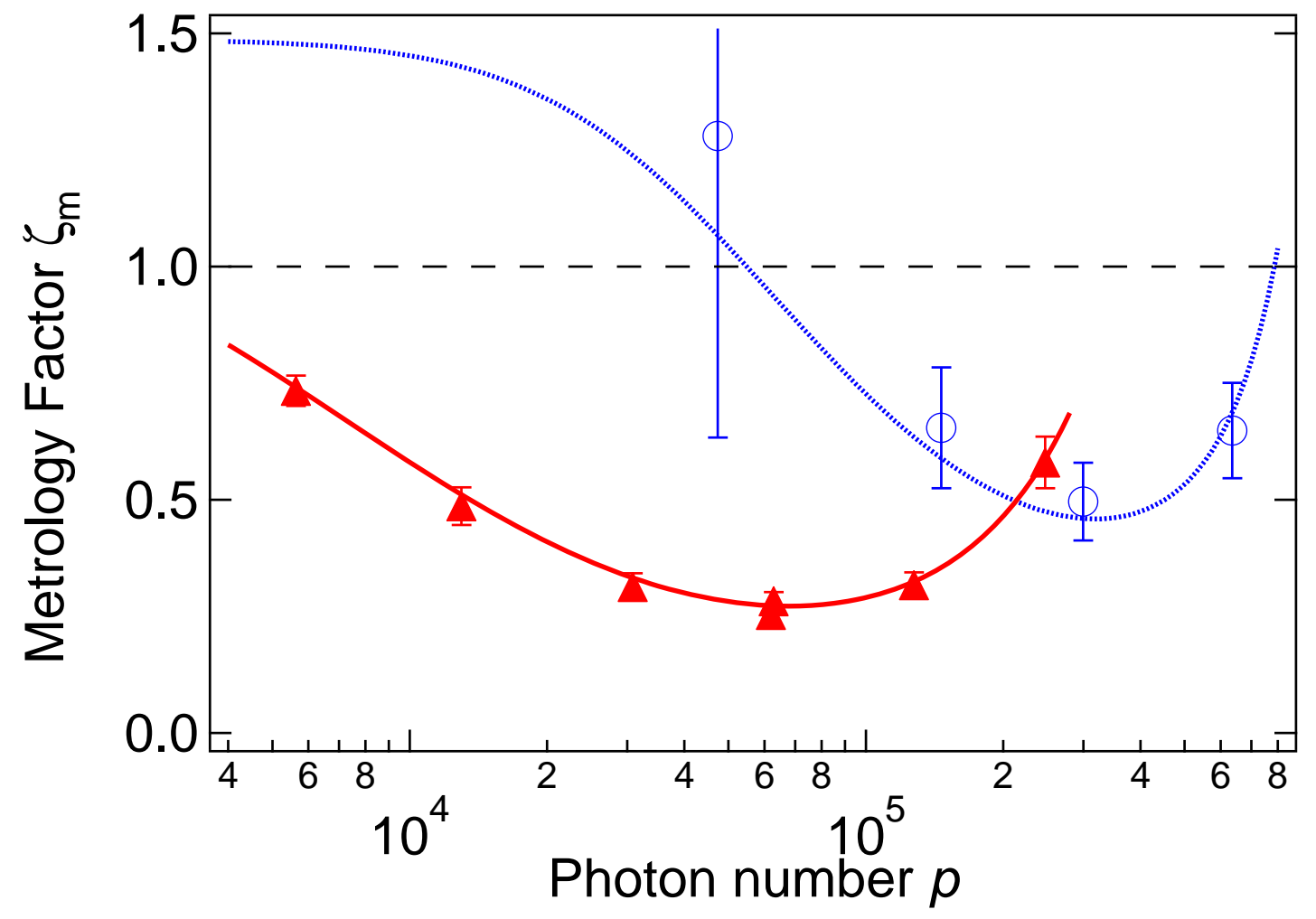

Figure 5. Comparison of metrology factors for conditional spin squeezing (open blue circles) and deterministic spin squeezing (solid red triangles), measured at effective atom number $N_{0} \approx 3 \times 10^{4}$. Curves are constructed from the fits to measured noise and contrast shown in the insets to Figs. 3 and 4.

deterministically prepare a spin-squeezed state allowing a 6.0(4) dB metrological improvement over the Standard Quantum Limit (SQL).

We have tested that both conditional and deterministic squeezing remain when the Ramsey clock sequence shown in Fig. 1D-F is inserted before the readout (at $X$ in Fig. 2(d)). So far, we have limited the precesion time to $70 \mu \mathrm{s}$, as observing the squeezing after longer time would require improved field stabilization. We would like to investigate the lifetime of the squeezing by applying it to the $\left|F=1, m_{F}=-1\right\rangle \rightarrow\left|F=2, m_{F}=1\right\rangle$ clock transition in magnetically trapped ${ }^{87} \mathrm{Rb}$, where long coherence times have already been demonstrated. ${ }^{30,31}$ Our method may also be extended beyond the microwave domain to benefit optical-transition atomic clocks. ${ }^{3-5}$

\section{APPENDIX A. "MAGIC POLARIZATION" TRAP}

To minimize inhomogeneous broadening of the clock transition, the trap light is elliptically polarized such that the vector light shift cancels, to lowest order, the differential scalar light shift of the clock states. Such a cancellation is possible because the vector light shift acts as an effective magnetic field ${ }^{32}$ and can be added to a real magnetic field to yield a quadratic Zeeman shift which, like the scalar light shift, is linear in the local intensity of trap light. In particular, in a magnetic field $B_{z}$ along the resonator axis, for an atom at potential $U<0$ in the dipole trap, the $\left|F=1, m_{F}=0\right\rangle \rightarrow\left|F=2, m_{F}=0\right\rangle$ transition frequency is shifted by

$$
\frac{\delta_{U}}{2 \pi}=-\frac{\omega_{\mathrm{HF}}}{\Delta} \frac{U}{h}+\beta\left(B_{z}+f b_{\sigma_{+}} \frac{U}{h}\right)^{2}
$$

where $\omega_{\mathrm{HF}}=2 \pi \times 6.835 \mathrm{GHz}$ is the ground-state hyperfine splitting; $\Delta^{-1}=\left(2 / \Delta_{2}+1 / \Delta_{1}\right) / 3$ in terms of the detunings $\Delta_{1}$ and $\Delta_{2}$ from the $D_{1}$ and $D_{2}$ lines; $\beta=574 \mathrm{~Hz} / \mathrm{G}^{2}$ sets the scale of the quadratic Zeeman shift; ${ }^{33}$ 
$f$ is the circular polarization fraction of the trap light; and the coefficient $b_{\sigma_{+}}=\left(\Delta_{1}^{-1}-\Delta_{2}^{-1}\right) \Delta \times h / 3 \mu_{B}=$ $0.061 \mathrm{G} / \mathrm{MHz}$ gives the effective magnetic field per unit trap depth for $\sigma_{+}$-polarized light. For a given field $B_{z}$, the broadening is minimized by choosing $f$ to place the minimum of the parabola described by Eq. 2 at the mean trapping potential $\langle U\rangle$ seen by the atoms.

For $B_{z}^{2}>\left(\omega_{\mathrm{HF}} / \Delta\right)\langle U\rangle / \beta$, the optimum circular polarization $f$ is inversely proportional to $B_{z}$, so that the residual broadening scales as $1 / B_{z}^{2}$. For our field of $B_{z}=5.6 \mathrm{G}$, we find an optimum circular polarization fraction $f=0.5(1)$, in agreement with the value calculated to minimize Eq. 2 at our trap depth $-U \approx 20 \mathrm{MHz}$. We measure a Ramsey coherence time of $10(2) \mathrm{ms}$, attributable to the residual broadening $\beta\left(f b_{\sigma_{+}} k T / h\right)^{2}$ associated with an axial temperature $k T / h \sim 5 \mathrm{MHz}$.

\section{ACKNOWLEDGMENTS}

We thank E. Polzik, J. Ye, J.K. Thompson, M.D. Lukin, and D. Stamper-Kurn for interesting discussions. This work was supported in part by the NSF, DARPA, and the NSF Center for Ultracold Atoms. M. S. acknowledges support from the Fannie and John Hertz Foundation Daniel Stroock Fellowship and NSF. I. D. L. acknowledges support from NSERC.

\section{REFERENCES}

1. G. Santarelli, P. Laurent, P. Lemonde, A. Clairon, A. G. Mann, S. Chang, A. N. Luiten, and C. Salomon, "Quantum projection noise in an atomic fountain: A high stability cesium frequency standard," Phys. Rev. Lett. 82, p. 4619, 1999.

2. M. Kitagawa and M. Ueda, "Squeezed spin states," Phys. Rev. A 47, p. 5138, 1993.

3. A. D. Ludlow, T. Zelevinsky, G. K. Campbell, S. Blatt, M. M. Boyd, M. H. G. de Miranda, M. J. Martin, J. W. Thomsen, S. M. Foreman, J. Ye, T. M. Fortier, J. E. Stalnaker, S. A. Diddams, Y. Le Coq, Z. W. Barber, N. Poli, N. D. Lemke, K. M. Beck, and C. W. Oates, "Sr Lattice Clock at 1 x 10-16 Fractional Uncertainty by Remote Optical Evaluation with a Ca Clock," Science 319(5871), pp. 1805-1808, 2008.

4. M. Takamoto, F.-L. Hong, R. Higashi, and H. Katori, "An optical lattice clock," Nature 435, pp. 321-324, 2005.

5. J. Ye, H. J. Kimble, and H. Katori, "Quantum state engineering and precision metrology using stateinsensitive light traps," Science 320, pp. 1734-1738, Jun 2008.

6. D. S. Durfee, Y. K. Shaham, and M. A. Kasevich, "Long-term stability of an area-reversible atominterferometer sagnac gyroscope," Phys. Rev. Lett. 97(24), p. 240801, 2006.

7. D. J. Wineland, J. J. Bollinger, W. M. Itano, F. L. Moore, and D. J. Heinzen, "Spin squeezing and reduced quantum noise in spectroscopy," Phys. Rev. A 46, p. R6797, 1992.

8. D. J. Wineland, J. J. Bollinger, W. M. Itano, and D. J. Heinzen, "Squeezed atomic states and projection noise in spectroscopy," Phys. Rev. A 50, p. R67, 1994.

9. A. S. Sørensen and K. Mølmer, "Entanglement and extreme spin squeezing," Phys. Rev. Lett. 86, pp. 44314434, 2001.

10. V. Meyer, M. A. Rowe, D. Kielpinski, C. A. Sackett, W. M. Itano, C. Monroe, and D. J. Wineland, "Experimental demonstration of entanglement-enhanced rotation angle estimation using trapped ions," Phys. Rev. Lett. 86, pp. 5870-5873, 2001.

11. A. André, A. S. Sørensen, and M. D. Lukin, "Stability of atomic clocks based on entangled atoms," Phys. Rev. Lett. 92, p. 230801, 2004.

12. A. Kuzmich, N. P. Bigelow, and L. Mandel, "Atomic quantum non-demolition measurements and squeezing," Europhys. Lett. 42(5), pp. 481-486, 1998.

13. I. Bouchoule and K. Mølmer, "Preparation of spin-squeezed atomic states by optical-phase-shift measurement," Phys. Rev. A 66, p. 043811, 2002.

14. L. B. Madsen and K. Mølmer, "Spin squeezing and precision probing with light and samples of atoms in the gaussian description," Phys. Rev. A 70, p. 052324, Nov 2004.

15. K. Hammerer, K. Mølmer, E. S. Polzik, and J. I. Cirac, "Light-matter quantum interface," Phys. Rev. A 70, p. 044304 , Oct 2004. 
16. J. Hald, J. L. Sørensen, C. Schori, and E. S. Polzik, "Spin squeezed atoms: A macroscopic entangled ensemble created by light," Phys. Rev. Lett. 83, p. 1319, 1999.

17. B. Julsgaard, A. Kozhekin, and E. S. Polzik, "Experimental long-lived entanglement of two macroscopic objects," Nature 413, pp. 400-403, 2001.

18. J. Appel, P. Windpassinger, D. Oblak, U. Hoff, N. Kjaergaard, and E. S. Polzik, "Mesoscopic atomic entanglement for precision measurements beyond the standard quantum limit," Proceedings of the National Academy of Sciences 106, pp. 10960-10965, 2009.

19. M. H. Schleier-Smith, I. D. Leroux, and V. Vuletić, "Reduced-quantum-uncertainty states of an ensemble of two-level atoms." quant-ph/arXiv0810.2582v2, 2009.

20. J. Estéve, C. Gross, A. Weller, S. Giovanazzi, and M. K. Oberthaler, "Squeezing and entanglement in a bose-einstein condensate," Nature 455, pp. 1216-1219, 2008.

21. G.-B. Jo, Y. Shin, S. Will, T. A. Pasquini, M. Saba, W. Ketterle, D. E. Pritchard, M. Vengalattore, and M. Prentiss, "Long phase coherence time and number squeezing of two Bose-Einstein condensates on an atom chip," Phys. Rev. Lett. 98, p. 030407, 2007.

22. D. Leibfried, M. D. Barrett, T. Schaetz, J. Britton, J. Chiaverini, W. M. Itano, J. D. Jost, C. Langer, and D. J. Wineland, "Toward Heisenberg-limited spectroscopy with multiparticle entangled states," Science 304, pp. 1476-1478, 2004.

23. D. Meiser, J. Ye, and M. J. Holland, "Spin squeezing in optical lattice clocks via lattice-based qnd measurements," New Journal of Physics 10, p. 073014 (17pp), 2008.

24. M. Saffman, D. Oblak, J. Appel, and E. S. Polzik, "Spin squeezing of atomic ensembles by multicolor quantum nondemolition measurements," Phys. Rev. A 79, p. 023831, 2009.

25. M. Ueda, T. Wakabayashi, and M. Kuwata-Gonokami, "Synchronous collapses and revivals of atomic dipole fluctuations and photon fano factor beyond the standard quantum limit," Phys. Rev. Lett. 76, pp. 20452048, Mar 1996.

26. M. Takeuchi, S. Ichihara, T. Takano, M. Kumakura, T. Yabuzaki, and Y. Takahashi, "Spin squeezing via one-axis twisting with coherent light," Phys. Rev. Lett. 94, p. 023003, 2005.

27. I. D. Leroux, M. H. Schleier-Smith, and V. Vuletić, 2009. To be published.

28. H. K. Cummins, G. Llewellyn, and J. A. Jones, "Tackling systematic errors in quantum logic gates with composite rotations," Phys. Rev. A 67, p. 042308, Apr 2003.

29. R. W. P. Drever, J. L. Hall, F. V. Kowalski, J. Hough, G. M. Ford, A. J. Munley, and H. Ward, "Laser phase and frequency stabilization using an optical resonator," Applied Physics B: Lasers and Optics 31, pp. 97-105, June 1983.

30. D. M. Harber, H. J. Lewandowski, J. M. McGuirk, and E. A. Cornell, "Effect of cold collisions on spin coherence and resonance shifts in a magnetically trapped ultracold gas," Phys. Rev. A 66, p. 053616, 2002.

31. P. Treutlein, P. Hommelhoff, T. Steinmetz, T. W. Hänsch, and J. Reichel, "Coherence in microchip traps," Phys. Rev. Lett. 92, p. 203005, 2004.

32. I. H. Deutsch and P. S. Jessen, "Quantum-state control in optical lattices," Phys. Rev. A 57, pp. 1972-1986, Mar 1998.

33. J. Vanier and C. Audoin, The Quantum Physics of Atomic Frequency Standards, Hilger, Philadelphia, 1989. 